\title{
The potential of FDG-PET in the detection of occult lymph node metastasis: importance of patient selection and reference standard
}

\author{
Remco de Bree $\cdot$ Otto S. Hoekstra
}

Received: 28 December 2012/ Accepted: 15 January 2013/Published online: 8 February 2013

(C) Springer-Verlag Berlin Heidelberg 2013

In patients with head and neck squamous cell carcinoma the presence of lymph node metastases is one of the most important prognostic factors. It is obvious that the patients with clinically manifest lymph node metastases require treatment of the neck. Therefore, the detection of lymph node metastases is of utmost importance. A meta-analysis showed that conventional imaging techniques, such as computed tomography (CT), magnetic resonance imaging (MRI), ultrasound (US) and especially US-guided fine needle aspiration cytology (USgFNAC), are more reliable than palpation [1]. Positron emission tomography (PET) imaging with F-18-fluorodeoxyglucose (18F-FDG) is a functional modality that has been used increasingly for staging head and neck cancer. Modern PET-CT imaging equipment combines both anatomic and functional imaging, potentially providing more accurate diagnosis and improved patient management. Here, the CT scan is used for attenuation correction of the PET images as well as for anatomic localisation; most scanners can provide state-ofthe-art contrast-enhanced CT (CECT) (and not just the low-dose CT [ld-CT] required for attenuation correction) together with PET in a single scan session.

Kyrzas et al. [2] found in another meta-analysis that 18F-FDG-PET has a good performance in the pre-

R. de Bree $(\bowtie)$

Department of Otolaryngology/Head and Neck Surgery, VU University Medical Center, De Boelelaan 1117, 1081 HV Amsterdam, The Netherlands

e-mail: r.debree@vumc.nl

R. de Bree

PO Box 7057, 1007 MB Amsterdam, The Netherlands

O. S. Hoekstra

Department of Radiology and Nuclear Medicine,

VU University Medical Center, Amsterdam, The Netherlands treatment evaluation of the presence of lymph node metastases in HNSSC patients: pooled sensitivity of $79 \%$ (95\% confidence interval (CI) 72-85\%) and pooled specificity of $86 \%$ (95\% CI 83-89\%). In studies in which both 18F-FDG-PET and conventional diagnostic tests were performed, sensitivity and specificity of 18F-FDG-PET were $80 \%(95 \% \mathrm{CI} 72-87 \%)$ and $86 \%(95 \% \mathrm{CI}$ 82-90\%), respectively, while for conventional diagnostic tests these figures were $75 \%$ (95\% CI 65-83\%) and $79 \%$ (95\% CI 72-85\%), respectively [2]. In 167 patients, Roh et al. [3] compared pre-operative PET or PETCT (only ld-CT) with CT or MRI for the detection of (occult and evident) lymph node metastases (per neck): sensitivity $90-91 \%$ and $76-77 \%$, and the specificity $87-88 \%$ and $81-83 \%$, respectively. No difference between PET only and PET-CT in accuracy was found [3].

However, the detection of occult lymph node metastases is the most important problem. The meta-analysis of Kyrzas et al. showed that 18F-FDG-PET detected only $50 \%$ (95\% CI 37-63\%) of the occult lymph node metastases, reiterating the inability of imaging tests to document microscopic disease. The specificity was $87 \%$ (CI 76-93\%)[2]. Krabbe et al. [4] found for the detection of occult metastases by FDG-PET a sensitivity of $50 \%$, a specificity of $97 \%$, negative predictive value of $88 \%$ and a positive predictive value of $80 \%$. In 18F-FDG-PET studies addressing HNSCC patients with a clinically negative neck sensitivity and specificity were quite variable. Brouwer et al. [5] showed that PET studies applying routine histopathological work-up reported much higher sensitivities to detect occult lymph node metastases than those using serial step sectioning and immunohistochemistry as the reference (gold) standard (67-100\% vs. 0-40\%) [5]. Routine histopathological examination can miss micrometastases in up to $15.2 \%$ [6] through which some 
false-negative findings may be incorrectly scored true negative. Since single tumour cells and micrometastases can also be missed by histopathological examination using step serial sectioning and immunohistochemistry, long term observation of the neck is even a better reference standard. It is important to realize that sensitivity is dependent on the reference standard used.

Additional explanations for heterogeneity may flow from differences in patient selection and inclusion criteria: not unexpectedly, studies reporting low sensitivity tend to have included patients with clinically as well as ultrasonographically negative necks, whereas higher sensitivities are reported if patients were included who were clinically negative but who more often had radiological evidence of lymph node metastases [7]. Therefore, it is of utmost importance that in clinical studies the definition of the clinically or radiologically negative neck is described in detail.

Jeong et al. [8] showed that PET-CT (ld-CT) was more accurate than PET alone and CECT alone for the conducting cervical lymph node evaluation in 47 HNSCC patients with and without clinical lymph node metastases who were scheduled for neck dissection(s): sensitivity of $91.8 \%$, $80.3 \%$ and $90.2 \%$, and specificity $98.8 \%, 92.8 \%$ and $93.9 \%$, respectively. Whereas ld-CT is used for attenuation correction, CECT may be helpful for the localisation of increased 18F-FDG uptake, e.g. differentiation between uptake in lymph nodes and brown fat, increasing 18F-FDGPET specificity. Ng et al. [9] showed that for the detection of subclinical lymph node metastases the visual correlation of 18F-FDG-PET with CE CT/MRI has been reported to be more accurate than 18F-FDG-PET alone. In 134 patients with oral squamous cell carcinoma they found a sensitivity of $51.4 \%$, which is increased to $57.1 \%$ after visual correlation with CT/MRI. This increment stemmed from the correction of false-negative 18F-FDG-PET results caused by necrotic nodes [9]. Ozer et al. [10] recently reported a sensitivity of $57 \%$ and specificity of $82 \%$ for the detection of occult metastasis by 18F-FDG-PET-CT in 112 patients with clinically negative neck according to physical examination, CT and/or MRI [10]. Unfortunately, CT scanning (ld-CT or CECT) was not defined. The study in the present issue of European Archives of Otorhinolaryngology shows a sensitivity of $84.2 \%$ and a specificity of $76.5 \%$ of $18 \mathrm{~F}-$ FDG-PET-CT in the detection of occult lymph node metastasis [11]. In interpreting these figures one should keep in mind the above discussed important aspects of chosen definition of clinically negative neck (patient selection), imaging technique (ld-CT or CECT) and reference standard (routine histopathological examination of neck dissection specimen, step serial sectioning and immunhistochemistry of all lymph nodes in neck dissection specimen or clinical follow-up).
Although 18F-FDG-PET and 18F-FDG-PET-CT may have the best accuracy for detecting occult cervical lymph node metastases in the clinical N0 neck, they are still not sufficiently reliable to avoid elective treatment of the neck. This can also be theoretically expected because of the limited resolution of the current PET scanners. Nevertheless, 18F-FDG-PET may provide important information about involvement of lymph nodes and be of value in treatment planning in head and neck cancer.

\section{References}

1. de Bondt RB, Nelemans PJ, Hofman PA, Casselman JW, Kremer B, van Engelshoven JM, Beets-Tan RG (2007) Detection of lymph node metastases in head and neck cancer: a meta-analysis comparing US, USgFNAC, CT and MR imaging. Eur J Radiol 64:266-272

2. Kyrzas PA, Evangolou E, Denaxa-Kyza D, Ionnidas JPA (2008) 18 F-Fluorodeoxyglucose positron emission tomography to evaluate cervical node metastases in patients with head and neck squamous cell carcinoma: a meta-analysis. J Natl Cancer Inst 100:712-720

3. Roh JL, Yeo NK, Kim JS, Lee JH, Cho JJ, Choi SH, Nam SY, Kim SY (2007) Utility of 2-[18F] fluoro-2-deoxy-D-glucose positron emission tomography and positron emission tomography/computed tomography imaging in the preoperative staging of head and neck squamous cell carcinoma. Oral Oncol 43:887-893

4. Krabbe CA, Dijkstra PU, Pruim J, van der Laan B, van der Wal JE, Gravendaal JP, Roodenburg JLN (2008) FDG PET in oral and oropharyngeal cancer. value for confirmation of NO neck and detection of occult metastases. Oral Oncol 44:31-36

5. Brouwer J, de Bree R, Comans EFI, Castelijns JA, Hoekstra OS, Leemans CR (2004) Positron emission tomography using [18F]fluorodeoxyglucose (FDG-PET) in the clinically negative neck; is it likely to be superior. Eur Arch Otorhinolaryngol 261:479-483

6. Rinaldo A, Devaney KO, Ferlito A (2004) Immunohistochemical studies in the identification of lymph node micrometastases in patients with squamous cell carcinoma of the head and neck. ORL J Otorhinolaryngol Relat Spec 66:38-41

7. Wensing BM, Vogel WV, Marres HAM, Merkx MAW, Postema EJ, Oyen WJG, van den Hoogen FJA (2006) FDG-PET in the clinically negative neck in oral squamous cell carcinoma. Laryngoscope 116:809-813

8. Jeong HS, Baek CH, Son YI, Chung MK, Lee DK, Choi JY, Kim BT, Kim HJ (2007) Use of integrated 18F-FDG PET/CT to improve the accuracy of initial cervical nodal evaluation in patients with head and neck squamous cell carcinoma. Head Neck 29:203-210

9. Ng SH, Yen TC, Chang JTC, Chan SC, Ko SF, Wang HM, Lee LY, Kang CJ, Wong AMC, Liao CT (2006) Prospective study of [18F]fluorodeoxuglucose positron emission tomography and magnetic resonance imaging in oral cavity squamous cell carcinoma with palpably negative neck. J Clin Oncol 27:4371-4376

10. Ozer E, Naiboğlu B, Meacham R, Ryoo C, Agrawal A, Schuller DE (2012) The value of PET/CT to assess clinically negative necks. Eur Arch Otorhinolaryngol 269:2411-2414

11. EAORL-D-12-00519 